\title{
Laparoscopic TME with APPEAR (Anterior and Perineal PlanE for ultra-low Anterior Resection of the Rectum) technique for distal rectal cancer
}

\author{
Saverio Di Palo • Paola De Nardi · Damiano Chiari • \\ Paolo Gazzetta $\cdot$ Carlo Staudacher
}

Received: 6 August 2012/ Accepted: 15 February 2013/Published online: 12 March 2013

(C) Springer Science+Business Media New York 2013

\begin{abstract}
Background Laparoscopic sphincter saving rectal resection for low rectal cancer is hampered by narrow pelvis and limitations of current stapling devices [1]. The APPEAR (Anterior Perineal PlanE for Ultra-low Anterior Resection of the Rectum) was proposed by Williams et al. [2, 3] as an alternative to the abdominal-perineal resection to perform very low rectal resection and anastomosis through a perineal wound. We adapted the original technique to the laparoscopic approach, avoiding any other abdominal incision.

Methods Between December 2011 and April 2012, five patients ( 2 females; median age 72 years (range 60-78)) with rectal cancer not involving the sphincters underwent laparoscopic total mesorectal excision (TME) with APPEAR. Mean distance of the tumor from anal verge was $3.2 \pm 1.1 \mathrm{~cm}$ (range 2-5).

Results All of the procedures were completed laparoscopically. All of the anastomoses were stapled, and a protective stoma was always constructed. The surgical specimens were retrieved from the perineal wound, and the stoma performed through one of the port sites, without any further abdominal incision. Mean operative time was $333 \pm 47$ min (range 295-405), postoperative stay $12 \pm 5$ days (range 6-17). Perineal wound infection was observed in three patients, two of whom also had anastomotic fistula, and was treated conservatively with prolonged suction drainage. Histological
\end{abstract}

Electronic supplementary material The online version of this article (doi:10.1007/s00464-013-2886-7) contains supplementary material, which is available to authorized users.

S. Di Palo · P. De Nardi $(\bowtie) \cdot$ D. Chiari · P. Gazzetta

C. Staudacher

Department of Surgery, San Raffaele Scientific Institute,

Via Olgettina 60, 20132 Milan, Italy

e-mail: denardi.paola@hsr.it examination showed three $\mathrm{pT} 3 \mathrm{~N}+$, one $\mathrm{T} 2 \mathrm{~N} 0$, and one complete response after neoadjuvant radiochemotherapy, with a mean distal clear margin of $1.27 \pm 0.5 \mathrm{~cm}$ (range 0.5-1.7). After a median follow-up of 9 months (range 8-12), one stoma reversal has been performed and the patient is fully continent.

Conclusions Our experience shows the feasibility of the APPEAR technique with laparoscopic TME, without any other abdominal incision. This technique offers advantage over the limitations of current laparoscopic stapling devices and their scanty maneuverability in the pelvis, allowing resection and anastomosis under direct vision, with adequate distal clearance, while sparing the anal sphincters.

Keywords Low rectal resection - Laparoscopy · Perineal approach

Disclosures S. Di Palo, P. De Nardi, D. Chiari, P. Gazzetta, and C. Staudacher have no conflict of interest.

\section{References}

1. Akiyoshi T, Kuroyanagi H, Oya M, Konishi T, Fukuda M, Fujimoto Y, Ueno M, Miyata S, Yamaguchi T (2009) Factors affecting the difficulty of laparoscopic total mesorectal excision with double stapling technique anastomosis for low rectal cancer. Surgery 146:483-489

2. Williams NS, Murphy J, Knowles CH (2008) Anterior Perineal PlanE for Ultra-low Anterior Resection of the rectum (The APPEAR Technique) a prospective clinical trial of a new procedure. Ann Surg 247:750-758

3. El-Gendy KA, Murphy J, Kullar NS, Chan CLH, Williams NS (2010) Anterior Perineal PlanE for Ultralow Anterior Resection of the Rectum (The APPEAR Technique): a video demonstration. Ann Surg Oncol 17:1357-1358 